\title{
ANOTHER PROOF OF THE THEOREMS ON THE EIGENVALUES OF A SQUARE QUATERNION MATRIX by YIK-HOI AU YEUNG
}

(Received 27 September, 1963)

1. Introduction. The nature of the eigenvalues of a square quaternion matrix had been considered by Lee [1] and Brenner [2]. In this paper the author gives another elementary proof of the theorems on the eigenvalues of a square quaternion matrix by considering the equation $G y=\mu \bar{y}$, where $G$ is an $n \times n$ complex matrix, $y$ is a non-zero vector in $C^{n}, \mu$ is a complex number, and $\bar{y}$ is the conjugate of $y$. The author wishes to thank Professor Y. C. Wong for his supervision during the preparation of this paper.

2. Notations. Let $R$ and $C$ be the field of real numbers and the field of complex numbers respectively, and $Q$ be the algebra of real quaternions. Then $Q$ has a base composed of four elements $e_{0}, e_{1}, e_{2}, e_{3}$ whose multiplication table is given by the following formulae:

$$
\begin{gathered}
e_{0} e_{\alpha}=e_{\alpha} e_{0}=e_{\alpha}, \quad e_{0}^{2}=e_{0}, \\
e_{\alpha}^{2}=-e_{0}, \quad e_{\alpha} e_{\beta}=-e_{\beta} e_{\alpha}=e_{\gamma},
\end{gathered}
$$

where $1 \leqq \alpha, \beta, \gamma \leqq 3$, and $(\alpha, \beta, \gamma)$ is a cyclic permutation of $(1,2,3)$. If $q \in Q$, then

$$
q=a_{0} e_{0}+a_{1} e_{1}+a_{2} e_{2}+a_{3} e_{3},
$$

where $a_{i} \in R(i=0,1,2,3)$. We shall identify $e_{0}$ and $e_{1}$ with 1 and $i(=\sqrt{ }-1)$ respectively, so that we can write $q=a_{0}+i a_{1}+e_{2}\left(a_{2}-i a_{3}\right)=\lambda+e_{2} \mu$, where $\lambda, \mu \in C$ (see Chevalley [3, pp. 16-17]). We define the norm of $q$ as the real number $\sum_{i=0}^{3} a_{i}^{2}$, and the trace of $q$ as $a_{0}$.

We regard $R^{n}$ and $C^{n}$ as vector spaces over $R$ and $C$, respectively, and $Q^{n}$ as a right vector space over $Q$.

3. The nature of the eigenvalues of a square quaternion matrix.

THEOREM 1. Let $F=G_{1}+e_{2} G_{2}$ be an $n \times n$ quaternion matrix, where $G_{1}$ and $G_{2}$ are complex matrices, and let

$$
G(\lambda) \equiv\left(\begin{array}{cc}
\bar{G}_{2} & G_{1}-\lambda I_{n} \\
-\bar{G}_{1}+\lambda I_{n} & G_{2}
\end{array}\right), \quad g(\lambda) \equiv|G(\lambda)|,
$$

where the bar denotes the complex conjugate, $|G(\lambda)|$ the determinant of the matrix $G(\lambda), I_{n}$ the $n \times n$ identity matrix, and $\lambda$ a complex variable.

(a) If $\alpha+i \beta+e_{2}(\gamma+i \delta)$ is any eigenvalue of $F$, then $\alpha+i k$, where $k^{2}=\beta^{2}+\gamma^{2}+\delta^{2}$, is a zero point of $g(\lambda)$.

(b) Conversely, if $\alpha+i k$ is any zero point of $g(\lambda)$, then $\alpha+i \beta+e_{2}(\gamma+i \delta)$, for any real numbers, $\beta, \gamma$ and $\delta$ such that $\beta^{2}+\gamma^{2}+\delta^{2}=k^{2}$, is an eigenvalue of $F$. 
Proof. As a first step in the proof of Theorem 1, we consider the following equation

$$
F x=x q,
$$

where $x=x_{1}+e_{2} x_{2} \neq 0$ with $x_{1}, x_{2} \in C^{n}$ and $q=\lambda+e_{2} \mu$ with $\lambda, \mu \in C$. Since

$$
\begin{aligned}
& F x=G_{1} x_{1}-\bar{G}_{2} x_{2}+e_{2}\left(G_{2} x_{1}+\bar{G}_{1} x_{2}\right), \\
& x q=x_{1} \lambda-\bar{x}_{2} \mu+e_{2}\left(x_{2} \lambda+\bar{x}_{1} \mu\right),
\end{aligned}
$$

equation (1) is equivalent to

which we can write as

$$
\left.\begin{array}{l}
G_{1} x_{1}-\bar{G}_{2} x_{2}=x_{1} \lambda-\bar{x}_{2} \mu \\
G_{2} x_{1}+\bar{G}_{1} x_{2}=x_{2} \lambda+\bar{x}_{1} \mu
\end{array}\right\}
$$

$$
\left(\begin{array}{cc}
G_{1}-\lambda I_{n} & -\bar{G}_{2} \\
G_{2} & \bar{G}_{1}-\lambda I_{n}
\end{array}\right)\left(\begin{array}{c}
x_{1} \\
x_{2}
\end{array}\right)=\mu\left(\begin{array}{c}
-\bar{x}_{2} \\
\bar{x}_{1}
\end{array}\right)
$$

But

$$
\begin{aligned}
\left(\begin{array}{cc}
G_{1}-\lambda I_{n} & -\bar{G}_{2} \\
G_{2} & \bar{G}_{1}-\lambda I_{n}
\end{array}\right)\left(\begin{array}{l}
x_{1} \\
x_{2}
\end{array}\right) & =\left(\begin{array}{cc}
G_{1}-\lambda I_{n} & -\bar{G}_{2} \\
G_{2} & \bar{G}_{1}-\lambda I_{n}
\end{array}\right)\left(\begin{array}{cc}
O & I_{n} \\
-I_{n} & O
\end{array}\right)\left(\begin{array}{c}
-x_{2} \\
x_{1}
\end{array}\right) \\
& =\left(\begin{array}{cc}
\bar{G}_{2} & G_{1}-\lambda I_{n} \\
-\bar{G}_{1}+\lambda I_{n} & G_{2}
\end{array}\right)\left(\begin{array}{c}
-x_{2} \\
x_{1}
\end{array}\right) .
\end{aligned}
$$

Therefore, equation (1) is equivalent to

$$
G(\lambda) y=\mu \bar{y},
$$

where

$$
G(\lambda)=\left(\begin{array}{cc}
\bar{G}_{2} & G_{1}-\lambda I_{n} \\
-\bar{G}_{1}+\lambda I_{n} & G_{2}
\end{array}\right), \quad y=\left(\begin{array}{c}
-x_{2} \\
x_{1}
\end{array}\right) \in C^{2 n} .
$$

Several lemmas are required to complete the proof of Theorem 1 .

Lemma 1. Let $U, V, W$ be $n \times n$ complex matrices and $\mu$ be a complex number; then

$$
\left|\begin{array}{cc}
\mu I_{n} & U \\
V & W
\end{array}\right|=|\mu W-V U| .
$$

Proof. If $\mu=0$, the result follows from Laplace's expansion.

If $\mu \neq 0$, then

$$
\left|\begin{array}{cc}
\mu I_{n} & U \\
V & W
\end{array}\right|=\left|\begin{array}{cc}
I_{n} & 0 \\
-\frac{1}{\mu} V & I_{n}
\end{array}\right|\left|\begin{array}{cc}
\mu I_{n} & U \\
V & W
\end{array}\right|=\left|\begin{array}{cc}
\mu I_{n} & U \\
O & W-\frac{1}{\mu} V U
\end{array}\right|,
$$

and again Laplace's expansion yields the result.

Lemma 2. Let $G=H_{1}+i H_{2}$, where $H_{1}$ and $H_{2}$ are $n \times n$ real matrices, and let

$$
h(\gamma, \delta) \equiv\left|\begin{array}{rr}
H_{1}-\gamma I_{n} & -H_{2}-\delta I_{n} \\
H_{2}-\delta I_{n} & H_{1}+\gamma I_{n}
\end{array}\right|, \quad p(t) \equiv\left|G \bar{G}-t I_{n}\right|,
$$


where $\gamma, \delta$ and t are real variables; then $\left(\gamma_{1}, \delta_{1}\right)$ is a zero point of $h(\gamma, \delta)$ if and only if $\gamma_{1}^{2}+\delta_{1}^{2}$ is a zero point of $p(t)$.

Proof.

$$
\begin{aligned}
h(\gamma, \delta) & =(-1)^{n}\left|\begin{array}{cc}
i I_{n} & I_{n} \\
O & I_{n}
\end{array}\right| \begin{array}{rr}
H_{1}-\gamma I_{n} & -H_{2}-\delta I_{n} \\
H_{2}-\delta I_{n} & H_{1}+\gamma I_{n}
\end{array}|| \begin{array}{cc}
i I_{n} & O \\
I_{n} & I_{n}
\end{array} \mid \\
& =(-1)^{n}\left|\begin{array}{cc}
2 \vec{\mu} I_{n} & \bar{G}+\bar{\mu} I_{n} \\
G+\bar{\mu} I_{n} & H_{1}+\gamma I_{n}
\end{array}\right|,
\end{aligned}
$$

where $\mu=\gamma+i \delta$. By Lemma 1, we have

$$
\begin{aligned}
h(\gamma, \delta) & =(-1)^{n}\left|2 \bar{\mu}\left(H_{1}+\gamma I_{n}\right)-\left(G+\bar{\mu} I_{n}\right)\left(\bar{G}+\bar{\mu} I_{n}\right)\right| \\
& =(-1)^{n}\left|2 \bar{\mu} H_{1}+2 \bar{\mu} \gamma I_{n}-G \bar{G}-2 \bar{\mu} H_{1}-\bar{\mu}^{2} I_{n}\right| \\
& =(-1)^{n}\left|\bar{\mu}(2 \gamma-\bar{\mu}) I_{n}-G \bar{G}\right| \\
& =(-1)^{n}\left|\left(\gamma^{2}+\delta^{2}\right) I_{n}-G \bar{G}\right| \\
& =(-1)^{2 n}\left|G \bar{G}-\left(\gamma^{2}+\delta^{2}\right) I_{n}\right| \\
& =p\left(\gamma^{2}+\delta^{2}\right) .
\end{aligned}
$$

Thus Lemma 2 is proved.

LEMma 3. Let $G=H_{1}+i H_{2}$, where $H_{1}$ and $H_{2}$ are $n \times n$ real matrices, and let $h(\gamma, \delta)$ and $p(t)$ be defined as in Lemma 2. Then the equation

$$
G y=\mu \bar{y},
$$

where $y=y_{1}+i y_{2} \neq 0$ with $y_{1}, y_{2} \in R^{n}$ and $\mu=\gamma+i \delta$ with $\gamma, \delta \in R$, is consistent if and only if $p\left(\gamma^{2}+\delta^{2}\right)=0$.

Proof. Since

$$
\begin{aligned}
G y & =H_{1} y_{1}-H_{2} y_{2}+i\left(H_{2} y_{1}+H_{1} y_{2}\right), \\
\mu \bar{y} & =\gamma y_{1}+\delta y_{2}+i\left(\delta y_{1}-\gamma y_{2}\right),
\end{aligned}
$$

equation (5) is equivalent to

$$
\left.\begin{array}{r}
\left(H_{1}-\gamma I_{n}\right) y_{1}+\left(-H_{2}-\delta I_{n}\right) y_{2}=O \\
\left(H_{2}-\delta I_{n}\right) y_{1}+\left(H_{1}+\gamma I_{n}\right) y_{2}=O
\end{array}\right\}
$$

where $y_{1}, y_{2}$ are not both zero. It follows from our definition of $h(\gamma, \delta)$ that equations (6) are consistent if and only if $(\gamma, \delta)$ is a zero point of $h(\gamma, \delta)$. Therefore, by Lemma 2, equations (6), and hence also equation (5), are consistent if and only if $p\left(\gamma^{2}+\delta^{2}\right)=0$. Thus Lemma 3 is proved.

Lemma 4. Let $G(\lambda)$ be defined as in Theorem 1 and let $p(\lambda, t)=\left|G(\lambda) \overline{G(\lambda)}-t I_{2 n}\right|$, where $t$ is a real variable. Then

(a) Equation (4), and hence also equation (1), and $\lambda=\alpha+i \beta, \mu=\gamma+i \delta$ are consistent if and only if $p\left(\alpha+i \beta, \gamma^{2}+\delta^{2}\right)=0$.

(b) $p\left(\alpha+i \beta, \gamma^{2}+\delta^{2}\right)=p\left(\alpha+i \beta_{1}, \gamma_{1}^{2}+\delta_{1}^{2}\right)$ for all real numbers $\beta_{1}, \gamma_{1}$ and $\delta_{1}$ such that $\beta_{1}^{2}+\gamma_{1}^{2}+\delta_{1}^{2}=\beta^{2}+\gamma^{2}+\delta^{2}$. 
Proof. (a) follows directly from Lemma 3. To prove (b), we note that for all real $\alpha, \beta, \gamma$ and $\delta$,

$$
\begin{aligned}
& p\left(\alpha+i \beta, \gamma^{2}+\delta^{2}\right)=\left|G(\alpha+i \beta) \overline{G(\alpha+i \beta)}-\left(\gamma^{2}+\delta^{2}\right) I_{2 n}\right| \\
& =\left|\begin{array}{cc}
\bar{G}_{2} G_{2}-G_{1} G_{1}+2 \alpha G_{1}-\left(a^{2}+\beta^{2}\right) I_{n} & \bar{G}_{2} \bar{G}_{1}+G_{1} \bar{G}_{2}-2 \alpha \bar{G}_{2} \\
-\left(\gamma^{2}+\delta^{2}\right) I_{n}^{\prime} & -\bar{G}_{1} \bar{G}_{1}+G_{2} \bar{G}_{2}+2 \alpha \bar{G}_{1}-\left(\alpha^{2}+\beta^{2}\right) I_{n} \\
-G_{2} G_{1}+2 \alpha G_{2} & -\left(\gamma^{2}+\delta^{2}\right) I_{n}
\end{array}\right| \\
& -\mid \begin{array}{ll}
\bar{G}_{2} G_{2}-G_{1} G_{1}+2 \alpha G_{1}-\alpha^{2} I_{n} & \bar{G}_{2} \bar{G}_{1}+G_{1} \bar{G}_{2}-2 \alpha \bar{G}_{2}
\end{array} \\
& =\mid \begin{array}{cc}
-\left(\beta^{2}+\gamma^{2}+\delta^{2}\right) I_{n} & \\
-\bar{G}_{1} G_{2}-G_{2} G_{1}+2 \alpha G_{2} & -\bar{G}_{1} \bar{G}_{1}+G_{2} \bar{G}_{2}+2 \alpha \bar{G}_{1}-\alpha^{2} I_{n}
\end{array} \\
& -\left(\beta^{2}+\gamma^{2}+\delta^{2}\right) I_{n} \\
& =\left|G(\alpha) \overline{G(\alpha)}-\left(\beta^{2}+\gamma^{2}+\delta^{2}\right) I_{2 n}\right|=p\left(\alpha, \beta^{2}+\gamma^{2}+\delta^{2}\right) \text {. }
\end{aligned}
$$

From this it follows that

$$
p\left(\alpha+i \beta, \gamma^{2}+\delta^{2}\right)=p\left(\alpha+i \beta_{1}, \gamma_{1}^{2}+\delta_{1}^{2}\right)
$$

for all real $\beta_{1}, \gamma_{1}$ and $\delta_{1}$ such that $\beta_{1}^{2}+\gamma_{1}^{2}+\delta_{1}^{2}=\beta^{2}+\gamma^{2}+\delta^{2}$.

Thus assertion (b) of Lemma 4 is proved.

The proof of Theorem 1 is now immediate. It follows from the definition that

$$
g(\lambda) \overline{g(\lambda)}=|G(\lambda) \overline{G(\lambda)}|=p(\lambda, 0)
$$

Therefore we have, by Lemma 4,

$$
g(\alpha+i k) \overline{g(\alpha+i k)}=p(\alpha+i k, 0)=p\left(\alpha+i \beta, \gamma^{2}+\delta^{2}\right),
$$

where $\beta, \gamma$ and $\delta$ are any real numbers such that $k^{2}=\beta^{2}+\gamma^{2}+\delta^{2}$. If $q=\alpha+i \beta+e_{2}(\gamma+i \delta)$ is any eigenvalue of $F$, then, by Lemma $4, p\left(\alpha+i \beta, \gamma^{2}+\delta^{2}\right)=0$. Therefore it follows from (8) that $\alpha+i k$ is a zero point of $g(\lambda)$. Thus assertion (a) of Theorem 1 is proved. Conversely, if $\alpha+i k$ is any zero point of $g(\lambda)$, then it follows from (8) that $p\left(\alpha+i \beta, \gamma^{2}+\delta^{2}\right)=0$ for any rea 1 $\beta, \gamma$ and $\delta$ such that $\beta^{2}+\gamma^{2}+\delta^{2}=k^{2}$. Therefore, by Lemma $4, \alpha+i \beta+e_{2}(\gamma+i \delta)$ is an eigenvalue of $F$. Thus assertion (b) of Theorem 1 is proved.

COROLlary 1. If $\tau$ is an eigenvalue of $F$ and $q$ is a quaternion such that $\tau$ and $q$ have equal norms and traces, then $q$ is an eigenvalue of $F$.

Proof. This is an immediate consequence of Theorem 1.

COROLLARY 2. If $q_{1}$ and $q_{2}$ are two quaternions having equal norms and traces, then there exists a quaternion $\sigma \neq 0$ such that $q_{2}=\sigma^{-1} q_{1} \sigma$.

Proof. Take $F=q_{1}$; then, since $q_{1} 1=1 q_{1}$, Corollary 2 follows from Corollary 1 . 
THEOREM 2. Let $F$ and $g(\lambda)$ be defined as in Theorem 1 ; then a complex number $\lambda$ is an eigenvalue of $F$ if and only if $\lambda$ is a zero point of $g(\lambda)$. And if $\tau$ is an eigenvalue of $F$, then $\sigma^{-1} \tau \sigma$ is also an eigenvalue of $F$ for all $\sigma \neq 0$ in $Q$. The class $\sigma^{-1} \tau \sigma$ contains just two complex numbers ( $\lambda$ and $\bar{\lambda}$ ).

Proof. Since $\tau$ and $\sigma^{-1} \tau \sigma$ have equal norms and traces, by Theorem 1, Corollaries 1 and 2 , Theorem 2 follows. we have

4. Remark. The polynomial $g(\lambda)$ defined in Theorem 1 has real coefficients. In fact,

$$
\begin{aligned}
g(\lambda) & =\left|\begin{array}{cc}
\bar{G}_{2} & G_{1}-\lambda I_{n} \\
-\bar{G}_{1}+\lambda I_{n} & G_{2}
\end{array}\right|=\left|\begin{array}{cc}
O & I_{n} \\
-I_{n} & O
\end{array}\right|\left|\begin{array}{cc}
\bar{G}_{2} & G_{1}-\lambda I_{n} \\
-\bar{G}_{1}+\lambda I_{n} & G_{2}
\end{array}\right|\left|\begin{array}{cc}
O & -I_{n} \\
I_{n} & O
\end{array}\right| \\
& =\left|\begin{array}{cc}
G_{2} & \bar{G}_{1}-\lambda I_{n} \\
-G_{1}+\lambda I_{n} & \bar{G}_{2}
\end{array}\right|=\bar{g}(\lambda) .
\end{aligned}
$$

\section{REFERENCES}

1. H. C. Lee, Eigenvalues and canonical forms of matrices with quaternion coefficients, Proc. Roy. Irish Acad. Sect. A, 52 (1949), 253-260.

2. J. L. Brenner, Matrices of quaternions, Pacific J. Math. 1 (1951), 329-335.

3. C. Chevalley, Theory of Lie groups (Princeton, 1946).

UNIVERSITY OF HONG KONG 